\title{
New Series Representation for the Trivariate Non-Central Chi-Squared Distribution
}

\author{
Prathapasinghe Dharmawansa, Member, IEEE, Nandana Rajatheva, Senior Member, IEEE, \\ and Chinthananda Tellambura, Senior Member, IEEE
}

\begin{abstract}
This paper derives a new infinite series representation for the trivariate Non-central chi-squared distribution when the underlying correlated Gaussian variables have a tridiagonal form of an inverse covariance matrix. The joint probability density function is derived using Miller's approach and Dougall's identity. Moreover, the trivariate cumulative distribution function (cdf) and characteristic function (chf) are also derived. Finally, the bivariate non-central chi-squared distribution and some known forms are shown to be special cases of the more general distribution. However, the derivation of non-central chi-squared distribution for an arbitrary covariance matrix seems intractable via Miller's approach. Two applications of the newly derived results are provided for performance analysis of multiple input multiple output (MIMO) systems with transmit antenna selection over a correlated Rician fading environment. Some numerical results are also presented to verify the accuracy of the analytical expressions.
\end{abstract}

Index Terms-Antenna selection, characteristic function (chf), MIMO systems, non-central chi-squared density, Rician fading, trivariate density.

\section{INTRODUCTION}

$\mathbf{T}$ HE $\chi^{2}$ and non-central $\chi^{2}$ distributions play a major role in the performance analysis of communication systems [1]- [5]. The generalized chi-squared distribution is analyzed in detail in [6]- [9]. Khaled and Williams [10] derive a relationship between non-central $\chi^{2}$ distribution and the distribution of a generalized Hermite quadratic form. The diagonal elements of a Wishart matrix are well known to have chi-squared distribution [11]. The joint probability density function (pdf) of the diagonal elements of a real central Wishart matrix (i.e., multivariate central $\chi^{2}$ distribution) is analyzed in [12][14]. The multivariate generalized Rayleigh pdf studied in [15] is also another form of multivariate central $\chi^{2}$ distribution. Nevertheless, the authors in [15], [16] assume a tridiagonal form of inverse correlation matrix for the underlying Gaussian variables to derive a closed-form solution for the generalized

Paper approved by M.-S. Alouini, the Editor for Modulation and Diversity Systems of the IEEE Communications Society. Manuscript received February 15, 2007; revised July 11, 2007 and December 10, 2007.

P. Dharmawansa was with the Telecommunications Field of Study, School of Engineering and Technology, Asian Institute of Technology, Thailand. He is now with the Department of Electronic and Computer Engineering, Hong Kong University of Science and Technology, Clear Water Bay, Kowloon, Hong Kong (e-mail: eesinghe@ust.hk).

N. Rajatheva is with the Telecommunications Field of Study, School of Engineering and Technology, Asian Institute of Technology, P. O. Box 4, Klong Luang, Pathumthani 12120, Thailand (e-mail: rajath@ait.ac.th).

C. Tellambura is with the Department of Electrical and Computer Engineering, University of Alberta, Edmonton, AB T6G 2V4, Canada (e-mail: chintha@ece.ualberta.ca).

Digital Object Identifier 10.1109/TCOMM.2009.03.070083
Rayleigh pdf. The trivariate generalized Rayleigh pdf for an arbitrary correlation matrix is given in [16]. In [17], Mallik derives multivariate Rayleigh and exponential distributions from correlated Gaussian random variables. Hagedorn et al. [11] derive a trivariate central chi square distribution from the diagonal elements of a complex Wishart matrix.

Miller's assumption of the tridiagonal form of inverse correlation matrix is significant since it leads to a closed-form solution for the multivariate Rayleigh pdf. Karagiannidis et al. [18], [19] extend Miller's result to $n$ variate Nakagami- $m$ distribution, which is also a form of a multivariate central $\chi^{2}$ pdf. We further derive quadrivariate Nakagami- $m$ distribution in [20] using Miller's method with the most general correlation matrix.

The Rice pdf is obviously closely coupled with noncentral $\chi^{2}$ distribution [21]. The multivariate non-central $\chi^{2}$ distribution can be thought of as a generalization of the multivariate Rician distribution. The bivariate Rician pdf is given in [22], [23] and [24]. In [25] the authors propose an infinite series representation involving modified Bessel functions of the first kind for the trivariate Rician distribution when the underlying Gaussian components have a tridiagonal form of inverse covariance matrix. The non-central Wishart distribution derived in [26] can be thought of as a more generalized version of the non-central distribution. Miller in [16] proposes an infinite series representation involving a modified Bessel function of the first kind for the pdf of the bivariate generalized Rician distribution. A search of the previous work related to multivariate distributions reveals that no joint pdf exists for trivariate non-central $\chi^{2}$ distribution.

For this reason, we propose a novel expression for the noncentral chi-squared pdf when the underlying Gaussian components have tridiagonal form of inverse covariance matrix. Our main derivation is inspired by Miller's approach [16] and by Dougall's theorem for a product of two ultraspherical polynomials [27]. Dougall's theorem which linearizes a product of two ultraspherical polynomials plays a major role in the density derivation. However, the derivation of noncentral trivariate pdf for an arbitrary covariance matrix seems intractable via Miller's approach. Finally, as applications of the newly derived pdf, we analyze the performance of multiple input multiple output (MIMO) systems with transmit antenna selection (TAS) by using orthogonal space-time block codes (OSTBC) in a correlated Rician fading environment.

This paper is organized as follows. Section II derives the generalized Rician distribution and trivariate non-central chi- 
squared distribution. Several simplifications related to previously known results are also discussed there. Section III deals with the derivation of cdf and chf with some simplifications. Applications of the pdf for MIMO systems with TAS are discussed in section IV. Section V concludes the paper.

\section{Derivation of Trivariate Non-CEntral $\chi^{2}$ DISTRIBUTION}

Let $\left\{\mathbf{X}_{1}, \mathbf{X}_{2}, \mathbf{X}_{3}\right\}$ be three nonzero mean Gaussian vectors with $E\left(\mathbf{X}_{i}\right)=\mathbf{a}$ and $\mathbf{X}_{i}=\left(x_{1 i} x_{2 i} \ldots \ldots x_{n i}\right)^{T}$ for all $1 \leq i \leq 3$. Here, $\mathbf{a}=\left(a_{1} a_{2} \ldots a_{n}\right)^{T}, E(\cdot)$ represents the mathematical expectation, and $(\cdot)^{T}$ denotes the transpose of a matrix. Let $\mathbf{V}_{j}=\left(\begin{array}{lll}x_{j 1} & x_{j 2} & x_{j 3}\end{array}\right), 1 \leq j \leq n$ be independent four-dimensional nonzero mean Gaussian vectors composed of the $j$ th components of $\mathbf{X}_{i}$. In this display, the columns are the $n$-dimensional Gaussian vectors

\begin{tabular}{c|ccc} 
& $\mathbf{X}_{1}$ & $\mathbf{X}_{2}$ & $\mathbf{X}_{3}$ \\
\hline $\mathbf{V}_{1}$ & $x_{11}$ & $x_{12}$ & $x_{13}$ \\
$\mathbf{V}_{2}$ & $x_{21}$ & $x_{22}$ & $x_{23}$ \\
& $\cdots$ & $\cdots$ & $\cdots$ \\
$\mathbf{V}_{n}$ & $x_{n, 1}$ & $x_{n, 2}$ & $x_{n, 3}$
\end{tabular}

and the rows $\mathbf{V}_{j}$ are independent from each other and have an identical covariance matrix $\mathbf{M}_{3}$. The inverse covariance matrix of $\mathbf{V}_{j}$ is

$$
\mathbf{W}_{3}=\mathbf{M}_{3}^{-1}=\left(\begin{array}{lll}
w_{11} & w_{12} & w_{13} \\
w_{12} & w_{22} & w_{23} \\
w_{13} & w_{23} & w_{33}
\end{array}\right) .
$$

The derivation of the joint pdf is analytically resolvable with Miller's approach if one or more off-diagonal elements of $\mathbf{W}_{3}$ are zero. The most general such realization is the tridiagonal form of a matrix or in other words, $w_{13}=0$. The exponential type covariance matrix is well known to give a tridiagonal form of inverse matrix [28]. This assumption is common for all the multivariate derivations given in [15], [18], [19].

The amplitudes $s_{i}=\left|\mathbf{X}_{i}\right|(1 \leq i \leq 3)$, being the square root of the sum of the squares of $n$ nonzero mean independent Gaussian random variables, are generalized Rician random variables. Here, $|\cdot|$ denotes the norm of a column vector. The joint pdf of $\left\{\mathbf{X}_{1}, \mathbf{X}_{2}, \mathbf{X}_{3}\right\}$ is clearly given by

$$
\begin{aligned}
f\left(\mathbf{X}_{1}, \mathbf{X}_{2}, \mathbf{X}_{3}\right) & =\prod_{j=1}^{n} f\left(\mathbf{V}_{\mathbf{j}}\right) \\
& =\frac{W_{3}^{\frac{n}{2}}}{(2 \pi)^{\frac{3 n}{2}}} \exp \left\{-\frac{1}{2} \sum_{j=1}^{n}\left(\mathbf{V}_{\mathbf{j}}-a_{j} \mathbf{1}_{3}\right) \mathbf{W}_{3}\left(\mathbf{V}_{\mathbf{j}}-a_{j} \mathbf{1}_{3}\right)^{T}\right\}
\end{aligned}
$$

where $\mathbf{1}_{3}=\left(\begin{array}{lll}1 & 1 & 1\end{array}\right)$ and $W_{3}$ denotes the determinant of square matrix W. Expanding the quadratic form in (3) and replacing the $\mathbf{V}_{j}$ 's by $\mathbf{X}_{i}$ (see the display in (1)), we find that

$$
\begin{aligned}
f\left(\mathbf{X}_{1}, \mathbf{X}_{2}, \mathbf{X}_{3}\right) & =\frac{W_{3}^{\frac{n}{2}}}{(2 \pi)^{\frac{3 n}{2}}} \exp \left\{-\frac{1}{2}\left(\sum_{i=0}^{3} w_{i i} s_{i}^{2}+w_{4} a^{2}\right)\right\} \\
& \times \exp \left\{\mathbf{X}_{1}^{T}\left(w_{1} \mathbf{a}-w_{12} \mathbf{X}_{2}\right)\right\} \exp \left(w_{2} \mathbf{X}_{2}^{T} \mathbf{a}\right) \\
& \times \exp \left\{\mathbf{X}_{3}^{T}\left(w_{3} \mathbf{a}-w_{23} \mathbf{X}_{2}\right)\right\}
\end{aligned}
$$

where $a^{2}=|\mathbf{a}|^{2}, w_{1}=w_{11}+w_{12}, w_{2}=w_{12}+w_{23}+$ $w_{22}, w_{3}=w_{23}+w_{33}$, and $w_{4}=w_{1}+w_{2}+w_{3}$. From this pdf (4), we need to integrate out $\mathbf{X}_{i}, 1 \leq i \leq 3$, subject to the constraints $s_{i}=\left|\mathbf{X}_{i}\right|$, which will yield the joint pdf of correlated generalized Rician variables $\left\{s_{1}, s_{2}, s_{3}\right\}$ [16]. Now, the joint pdf can be written as

$$
\begin{aligned}
f\left(s_{1}, s_{2}, s_{3}\right) & =\frac{W_{3}^{\frac{n}{2}}}{(2 \pi)^{\frac{3 n}{2}}} \exp \left\{-\frac{1}{2}\left(\sum_{i=1}^{3} w_{i i} s_{i}^{2}+w_{4} a^{2}\right)\right\} \\
& \times \int_{\left|\mathbf{X}_{1}\right|=s_{1}} \exp \left\{\mathbf{X}_{1}^{T}\left(w_{1} \mathbf{a}-w_{12} \mathbf{X}_{2}\right)\right\} d \sigma_{x_{1}} \\
& \times \int_{\left|\mathbf{X}_{3}\right|=s_{3}} \exp \left\{\mathbf{X}_{3}^{T}\left(w_{3} \mathbf{a}-w_{23} \mathbf{X}_{2}\right)\right\} d \sigma_{x_{3}} \\
& \times \int_{\left|\mathbf{X}_{2}\right|=s_{2}} \exp \left(w_{2} \mathbf{X}_{2}^{T} \mathbf{a}\right) d \sigma_{x_{2}},
\end{aligned}
$$

where $d \sigma_{x_{i}}, 1 \leq i \leq 3$ are the elements of surface area. The first integral in (5) can be evaluated as [16, eq.2.2.9]

$$
\begin{aligned}
& \int_{\left|\mathbf{X}_{1}\right|=s_{1}} \exp \left\{\mathbf{X}_{1}^{T}\left(w_{1} \mathbf{a}-w_{12} \mathbf{X}_{2}\right)\right\} d \sigma_{x_{1}} \\
&=\left(2 \pi s_{1}\right)^{\frac{n}{2}}\left|w_{1} \mathbf{a}-w_{12} \mathbf{X}_{2}\right|^{\frac{2-n}{2}} \\
& \times I_{\frac{n-2}{2}}\left(s_{1}\left|w_{1} \mathbf{a}-w_{12} \mathbf{X}_{2}\right|\right),
\end{aligned}
$$

where $I_{n}(z)$ is the $n$th order modified Bessel function of the first kind [29], and the second integral follows the same form. Furthermore, the right side of (6) can be written by using the generalized Neumann addition formula [29] when $n>2$ as

$$
\begin{gathered}
\left(2 \pi s_{1}\right)^{\frac{n}{2}}\left|w_{1} \mathbf{a}-w_{12} \mathbf{X}_{2}\right|^{\frac{2-n}{2}} I_{\frac{n-2}{2}}\left(s_{1} \mid w_{1} \mathbf{a}-w_{12} \mathbf{X}_{2}\right) \\
=\frac{\left(2 \pi s_{1}\right)^{\frac{n}{2}} 2^{\frac{n-2}{2}} \Gamma\left(\frac{n-2}{2}\right) s_{1}}{\left(w_{1} w_{12} a s_{2}\right)^{\frac{n-2}{2}}} \sum_{k=0}^{\infty}(-1)^{k} \nu_{k} \\
\quad \times I_{\nu_{k}}\left(a w_{1} s_{1}\right) I_{\nu_{k}}\left(w_{12} s_{1} s_{2}\right) C_{k}^{\frac{n-2}{2}}(\cos \theta)
\end{gathered}
$$

where $\nu_{k}=\frac{n}{2}+k-1, \Gamma(z)=\int_{0}^{\infty} t^{z-1} \exp (-t), z>0$ is the Gamma function [30], $C_{n}^{\lambda}(z)$ denotes the ultraspherical polynomials [30] defined as

$$
\left(1-2 z r+r^{2}\right)^{-\lambda}=\sum_{n=0}^{\infty} C_{n}^{\lambda}(z) r^{n}, \quad \lambda>-\frac{1}{2}
$$

$$
\begin{aligned}
f\left(s_{1}, s_{2}, s_{3}\right)= & \frac{W_{3}^{\frac{n}{2}} 2^{n-2} \Gamma^{2}\left(\frac{n-2}{2}\right) s_{1} s_{3}}{(2 \pi)^{\frac{n}{2}}\left(w_{1} w_{12} w_{23} w_{3}\right)^{\frac{n-2}{2}}\left(a s_{2}\right)^{n-2}} \exp \left\{-\frac{1}{2}\left(\sum_{i=1}^{3} w_{i i} s_{i}^{2}+w_{4} a^{2}\right)\right\} \sum_{k=0}^{\infty} \sum_{l=0}^{\infty}(-1)^{k+l} \nu_{k} \nu_{l} I_{\nu_{k}}\left(a w_{1} s_{1}\right) \\
& I_{\nu_{k}}\left(w_{12} s_{1} s_{2}\right) I_{\nu_{l}}\left(a w_{3} s_{3}\right) I_{\nu_{l}}\left(w_{23} s_{2} s_{3}\right) \int_{\left|\mathbf{X}_{2}\right|=s_{2}} \exp \left(w_{2} \mathbf{X}_{2}^{T} \mathbf{a}\right) C_{k}^{\frac{n-2}{2}}(\cos \theta) C_{l}^{\frac{n-2}{2}}(\cos \theta) d \sigma_{x_{2}} .
\end{aligned}
$$


and $\theta$ is the angle between the vectors $\mathbf{a}$ and $\mathbf{X}_{2}$. Following (7), (5) can be written as given at the bottom of the previous page. The product of two ultraspherical polynomials can be written by using the Dougall's identity, given in [27, eq.6.8.4] as

$$
C_{p}^{\lambda}(z) C_{q}^{\lambda}(z)=\sum_{m=0}^{\min (p, q)} A(m, p, q) C_{p+q-2 n}^{\lambda}(z),
$$

where $A(m, p, q)$ is given by

$$
A(m, p, q)=\frac{\left(\mu_{\lambda}-m\right)(\lambda)_{m}(\lambda)_{p-m}(\lambda)_{q-m}(2 \lambda)_{\mu_{0}}\left(\mu_{0}-m\right) !}{\mu_{\lambda} m !(p-m) !(q-m) !(\lambda)_{\mu_{0}}(2 \lambda)_{\mu_{0}-m}}
$$

with $\mu_{k}=p+q+k-m,(\lambda)_{n}=\frac{\Gamma(\lambda+n)}{\Gamma(\lambda)}$ denoting the Pochhammer symbol, and $\min (p, q)$ selecting the minimum of $p, q$. We use Dougall's identity, given in (9), to yield (10). The integral in (10) can be solved by using [16, eq.2.2.26] to give

$$
\begin{aligned}
\int_{\left|\mathbf{X}_{2}\right|=s_{2}} \exp \left(w_{2} \mathbf{X}_{2}^{T} \mathbf{a}\right) C_{\eta_{k l}}^{\frac{n-2}{2}}(\cos \theta) d \sigma_{x_{2}} & \\
= & \frac{(2 \pi)^{\frac{n}{2}} s_{2}^{n-1}\left(\begin{array}{c}
n+\eta_{k l}-3 \\
n-3
\end{array}\right)}{\left(a w_{2} s_{2}\right)^{\frac{n-2}{2}}} I_{\nu_{\eta_{k l}}}\left(a w_{2} s_{2}\right)
\end{aligned}
$$

where $\eta_{k l}=k+l-2 m$ and $\left(\begin{array}{c}n \\ r\end{array}\right)=\frac{n !}{r !(n-r) !}$. Substituting the above integral in (10) and after some algebraic manipulations, the joint pdf of the multivariate generalized Rician distribution for $n>2$ can be written as shown in (11). The case when $n=2$ is given in [25, eq.3].

In order to derive the trivariate non-central $\chi^{2}$ pdf, the following variable transformations are introduced in (11), $r_{1}=s_{1}^{2}, r_{2}=s_{2}^{2}, r_{3}=s_{3}^{2}$. Now, it is clear that $\left\{r_{1}, r_{2}, r_{3}\right\}$ represent the correlated non-central $\chi^{2}$ variables. After some algebraic manipulation, the trivariate non-central $\chi^{2}$ pdf can be written as given in (12). To the best of the authors' knowledge, (12) is a novel result. Even though (12) is not valid for $n=2$, the degenerated cases of (12) are valid for all $n \geq 2$ as shown below. Moreover, if a given covariance matrix does not match with the criteria mentioned above, we can use Green's matrix approach, given in [19], to approximately find a matrix with a tridiagonal form of inverse. Next, several simplifications of (12) are given.

\section{A. Independent non-central $\chi^{2}$ case}

$\mathbf{W}_{3}$ is obviously a diagonal matrix with the elements $\left\{w_{11}, w_{22}, w_{33}\right\}$ under this scenario. Since all the off-diagonal elements are zero, the following important limit involving the Bessel functions is obtained:

$$
\lim _{w_{i i+1} \rightarrow 0} \frac{I_{\frac{n}{2}+k-1}\left(w_{i i+1} \sqrt{r_{i} r_{i+1}}\right)}{w_{i i+1}^{\frac{n-2}{2}}}=\frac{\left(\frac{\sqrt{r_{i} r_{i+1}}}{2}\right)^{\frac{n}{2}-1}}{\Gamma\left(\frac{n}{2}\right)}
$$

which is valid for $i=1,2$ if $k=0$. Using (13) in (12) and after algebraic rearrangements, the joint pdf is obtained as

$$
\begin{aligned}
g\left(r_{1}, r_{2}, r_{3}\right)=\prod_{i=1}^{3} \frac{w_{i i}}{2}\left(\frac{r_{i}}{a^{2}}\right)^{\frac{n-2}{4}} & \exp \left\{-\frac{w_{i i}}{2}\left(r_{i}+a^{2}\right)\right\} \\
& \times I_{\frac{n}{2}-1}\left(a w_{i i} \sqrt{r_{i}}\right) .
\end{aligned}
$$

The extension for central $\chi^{2}$ distribution follows from (14).

\section{B. Bivariate non-central $\chi^{2}$ distribution}

If $\left\{r_{1}, r_{2}\right\}$ are independent from $r_{3}$, the trivariate pdf can be written as a product of bivariate and univariate pdf's. Equating $w_{23}$ to zero and using the limit (13) with $l=0$ followed by some manipulations, the bivariate pdf is obtained as

$$
\begin{aligned}
g\left(r_{1}, r_{2}\right)= & \frac{W_{2}^{\frac{n}{2}} 2^{\frac{n}{2}} \Gamma\left(\frac{n-2}{2}\right)}{8\left(w_{1} w_{2} w_{12}\right)^{\frac{n-2}{2}}(a)^{n-2}} e^{-\frac{1}{2}\left(\sum_{i=1}^{2} w_{i i} r_{i}+w_{i} a^{2}\right)} \\
& \times \sum_{k=0}^{\infty}(-1)^{k}\left(\begin{array}{c}
n+k-3 \\
n-3
\end{array}\right) \nu_{k} I_{\nu_{k}}\left(a w_{1} \sqrt{r_{1}}\right) \\
& \times I_{\nu_{k}}\left(w_{12} \sqrt{r_{1} r_{2}}\right) I_{\nu_{k}}\left(a w_{2} \sqrt{r_{2}}\right),
\end{aligned}
$$

$$
\begin{aligned}
& f\left(s_{1}, s_{2}, s_{3}\right)=\frac{W_{3}^{\frac{n}{2}} 2^{n-2} \Gamma^{2}\left(\frac{n-2}{2}\right) s_{1} s_{3}}{(2 \pi)^{\frac{n}{2}}\left(w_{1} w_{12} w_{23} w_{3}\right)^{\frac{n-2}{2}}\left(a s_{2}\right)^{n-2}} \exp \left\{-\frac{1}{2}\left(\sum_{i=1}^{3} w_{i i} s_{i}^{2}+w_{4} a^{2}\right)\right\} \sum_{k=0}^{\infty} \sum_{l=0}^{\infty} \sum_{m=0}^{\min (k, l)}(-1)^{k+l} \nu_{k} \nu_{l} A(m, k, l) \\
& \times I_{\nu_{k}}\left(a w_{1} s_{1}\right) I_{\nu_{k}}\left(w_{12} s_{1} s_{2}\right) I_{\nu_{l}}\left(a w_{3} s_{3}\right) I_{\nu_{l}}\left(w_{23} s_{2} s_{3}\right) \int_{\left|\mathbf{X}_{2}\right|=s_{2}} \exp \left(w_{2} \mathbf{X}_{2}^{T} \mathbf{a}\right) C_{k+l-2 m}^{\frac{n-2}{2}}(\cos \theta) d \sigma_{x_{2}} . \\
& f\left(s_{1}, s_{2}, s_{3}\right)=\frac{W_{3}^{\frac{n}{2}} 2^{n-2} \Gamma^{2}\left(\frac{n-2}{2}\right) s_{1} s_{2} s_{3}}{\left(w_{1} w_{2} w_{3} w_{12} w_{23} a s_{2}\right)^{\frac{n-2}{2}}(a)^{n-2}} \exp \left\{-\frac{1}{2}\left(\sum_{i=1}^{3} w_{i i} s_{i}^{2}+w_{4} a^{2}\right)\right\} \sum_{k=0}^{\infty} \sum_{l=0}^{\infty} \sum_{m=0}^{\min (k, l)}(-1)^{k+l} \nu_{k} \nu_{l}\left(\begin{array}{c}
n+\eta_{k l}-3 \\
n-3
\end{array}\right) \\
& \times A(m, k, l) I_{\nu_{k}}\left(a w_{1} s_{1}\right) I_{\nu_{k}}\left(w_{12} s_{1} s_{2}\right) I_{\nu_{l}}\left(a w_{3} s_{3}\right) I_{\nu_{l}}\left(w_{23} s_{2} s_{3}\right) I_{\nu_{\eta_{k l}}}\left(a w_{2} s_{2}\right) \text {. } \\
& g\left(r_{1}, r_{2}, r_{3}\right)=\frac{W_{3}^{\frac{n}{2}} 2^{n} \Gamma^{2}\left(\frac{n-2}{2}\right)}{32\left(w_{1} w_{2} w_{3} w_{12} w_{23}\right)^{\frac{n-2}{2}}(a)^{\frac{3 n}{2}-3}\left(\sqrt{r_{2}}\right)^{\frac{n-2}{2}}} \exp \left\{-\frac{1}{2}\left(\sum_{i=1}^{3} w_{i i} r_{i}+w_{4} a^{2}\right)\right\} \sum_{k=0}^{\infty} \sum_{l=0}^{\infty} \sum_{m=0}^{\min (k, l)}(-1)^{k+l} \nu_{k} \nu_{l} \\
& \times A(m, k, l)\left(\begin{array}{c}
n+\eta_{k l}-3 \\
n-3
\end{array}\right) I_{\nu_{k}}\left(a w_{1} \sqrt{r_{1}}\right) I_{\nu_{k}}\left(w_{12} \sqrt{r_{1} r_{2}}\right) I_{\nu_{l}}\left(a w_{3} \sqrt{r_{3}}\right) I_{\nu_{l}}\left(w_{23} \sqrt{r_{2} r_{3}}\right) I_{\nu_{\eta_{k l}}}\left(a w_{2} \sqrt{r_{2}}\right) .
\end{aligned}
$$


TABLE I

Number of Terms Needed in (12) to Achieve Five Significant Figure Accuracy.

\begin{tabular}{|c|c|c|c|c|}
\hline$a$ & $\rho$ & $r_{1}=1.5, r_{2}=2.3, r_{3}=3.4$ & $r_{1}=3.7, r_{2}=4.2, r_{3}=3.8$ & $r_{1}=2.8, r_{2}=5.1, r_{3}=4.6$ \\
\hline 1 & \multirow{2}{*}{0.1} & $K=3, L=3$ & $K=3, L=3$ & $K=3, L=3$ \\
3 & & $K=4, L=4$ & $K=5, L=4$ & $K=5, L=5$ \\
\hline 1 & \multirow{2}{*}{0.4} & $K=4, L=3$ & $K=4, L=4$ & $K=4, L=4$ \\
3 & & $K=4, L=4$ & $K=5, L=5$ & $K=6, L=6$ \\
\hline 1 & \multirow{2}{*}{0.8} & $K=4, L=4$ & $K=4, L=4$ & $K=4, L=4$ \\
3 & & $K=5, L=5$ & $K=6, L=6$ & $K=6, L=6$ \\
\hline
\end{tabular}

TABLE II

Number of Terms Needed in (12) to Achieve Five Significant FIGURE ACCURACY WHEN $r_{1}=r_{2}=r_{3}=r$.

\begin{tabular}{|c|c|c|c|c|}
\hline$a$ & $\rho$ & $r=1$ & $r=2$ & $r=5$ \\
\hline 1 & \multirow{2}{*}{0.1} & $K=2, L=2$ & $K=3, L=3$ & $K=3, L=3$ \\
3 & & $K=3, L=3$ & $K=4, L=4$ & $K=4, L=4$ \\
\hline 1 & \multirow{2}{*}{0.4} & $K=3, L=3$ & $K=4, L=4$ & $K=4, L=4$ \\
3 & & $K=3, L=3$ & $K=5, L=5$ & $K=6, L=6$ \\
\hline 1 & \multirow{2}{*}{0.8} & $K=3, L=3$ & $K=4, L=4$ & $K=5, L=5$ \\
3 & & $K=4, L=4$ & $K=6, L=6$ & $K=6, L=6$ \\
\hline
\end{tabular}

where $W_{2}$ denotes the determinant of $2 \times 2$ inverse covariance matrix $\mathbf{W}_{2}$. Equation (15) is equivalent to Miller's previously published result [16, eq.2.2.18].

Now let us consider the truncation of the series (12) because for all practical numerical calculations the infinite series are truncated to limited number of terms. This operation introduces a truncation error. Suppose that the series (12) is limited to $K$ and $L$ terms in the variables $k$ and $l$ respectively. Hence the truncation error can be expressed as

$$
E_{T R(p d f)}=\sum_{k=0}^{K-1} \sum_{l=L}^{\infty} \sum_{m=0}^{\min (k, l)} R_{\epsilon}+\sum_{k=K}^{\infty} \sum_{l=0}^{\infty} \sum_{m=0}^{\min (k, l)} R_{\epsilon}
$$

where

$$
\begin{aligned}
R_{\epsilon}= & (-1)^{k+l} \frac{W_{3}^{\frac{n}{2}} 2^{n} \Gamma^{2}\left(\frac{n-2}{2}\right) e^{-\frac{1}{2}\left(\sum_{i=1}^{3} w_{i i} r_{i}+w_{4} a^{2}\right)}}{32\left(w_{1} w_{2} w_{3} w_{12} w_{23}\right)^{\frac{n-2}{2}} a^{\frac{3 n}{2}-3}\left(\sqrt{r_{2}}\right)^{\frac{n-2}{2}}} \\
& \times A(m, k, l)\left(\begin{array}{c}
n+\eta_{k l}-3 \\
n-2
\end{array}\right) \nu_{k} \nu_{l} I_{\nu_{k}}\left(a w_{1} \sqrt{r_{1}}\right) \\
& \times I_{\nu_{k}}\left(w_{12} \sqrt{r_{1} r_{2}}\right) I_{\nu_{l}}\left(a w_{3} \sqrt{r_{3}}\right) I_{\nu_{l}}\left(w_{23} \sqrt{r_{2} r_{3}}\right) \\
& \times I_{\nu_{\eta_{k l}}}\left(a w_{2} \sqrt{r_{2}}\right) .
\end{aligned}
$$

Furthermore, to shed some light on the convergence of (12), the number of terms needed in (12) to achieve five significant figure accuracy is given in Table 1 and Table II. The exponential covariance model proposed by Aalo [28] is used in the calculations.

\section{CDF AND ChF of the Trivariate Distribution}

\section{A. Cumulative Distribution Function}

The trivariate cdf is, by definition [31],

$$
G\left(r_{1}, r_{2}, r_{3}\right)=\int_{0}^{r_{1}} \int_{0}^{r_{2}} \int_{0}^{r_{3}} g\left(y_{1}, y_{2}, y_{3}\right) d y_{1} d y_{2} d y_{3} .
$$

Substituting (12) in (16) and expanding each Bessel function term with its infinite series representation, followed by termby-term integration, the cdf of trivariate non-central $\chi^{2}$ distribution is obtained as given in (17), where $\lambda_{1}=2 i_{1}+k$, $\lambda_{2}=2 i_{5}+k+l-2 m, \lambda_{3}=2 i_{3}+l, \lambda_{4}=2 i_{2}+k, \lambda_{5}=2 i_{4}+l$, $\delta_{1}=i_{1}+i_{2}+k+\frac{n}{2}, \delta_{2}=i_{2}+i_{4}+i_{5}+k+\frac{n}{2}-m$, $\delta_{3}=i_{3}+i_{4}+l+\frac{n}{2}$, and $\gamma(a, z)=\int_{0}^{z} t^{a-1} \exp (-t) d t$ is the incomplete gamma function.

Moreover, if $n>2$ is an even integer, the alternative closed form expression given in [21] can be used instead of the incomplete gamma function. The simplifications for special cases are straightforward with (17).

\section{B. Characteristic Function}

The joint chf is defined as [31]

$$
\psi\left(v_{1}, v_{2}, v_{3}\right)=E\left\{\exp \left(v_{1} r_{1} j+v_{2} r_{2} j+v_{3} r_{3} j\right)\right\},
$$

where $j=\sqrt{-1}$. As with the cdf derivation, we encounter integrals of the form

$$
\int_{0}^{\infty} z^{\nu-1} \exp (-[p+j q] z) d z,
$$

which can be solved by using [31, eq.3.381.5] to yield the chf as shown in (19).

The bivariate generalization is straightforward with (19). If all $\left\{r_{1}, r_{2}, r_{3}\right\}$ are independent (i.e., $w_{12}=w_{23}=0, i_{2}=$ $i_{4}=k=l=0$ in (19)), then the joint chf can be written as a product of the individual chfs. Thus, we arrive at a product

$$
\begin{aligned}
G\left(r_{1}, r_{2}, r_{3}\right)= & W_{3}^{\frac{n}{2}} \Gamma^{2}\left(\frac{n-2}{2}\right) \exp \left(-\frac{a^{2} w_{4}}{2}\right) \sum_{k, l=0}^{\infty} \sum_{m=0}^{\min (k, l)}(-1)^{k+l} A(m, k, l) \nu_{k} \nu_{l}\left(\begin{array}{c}
n+\eta_{k l}-3 \\
n-3
\end{array}\right) \\
& \times \sum_{i_{1}, i_{2}, i_{3}, i_{4}, i_{5}=0}^{\infty} \frac{\gamma\left(\delta_{1}, \frac{w_{11}}{2} r_{1}\right) \gamma\left(\delta_{2}, \frac{w_{22}}{2} r_{2}\right) \gamma\left(\delta_{3}, \frac{w_{33}}{2} r_{3}\right)}{2^{\frac{1}{2}\left(\lambda_{1}+\lambda_{2}+\lambda_{3}\right)} i_{1} ! i_{2} ! i_{3} ! i_{4} ! i_{5} ! \Gamma\left(i_{1}+\frac{n}{2}+k\right) \Gamma\left(i_{2}+\frac{n}{2}+k\right) \Gamma\left(i_{3}+\frac{n}{2}+l\right)} \\
& \times \frac{a^{\lambda_{1}+\lambda_{2}+\lambda_{3}} w_{1}^{\lambda_{1}} w_{2}^{\lambda_{2}} w_{3}^{\lambda_{3}} w_{12}^{\lambda_{4}} w_{23}^{\lambda_{5}}}{\Gamma\left(i_{4}+\frac{n}{2}+l\right) \Gamma\left(i_{5}+\frac{n}{2}+k+l-2 m\right) w_{11}^{\delta_{1}} w_{22}^{\delta_{2}} w_{33}^{\delta_{3}}}
\end{aligned}
$$


TABLE III

Number of Terms NeEded in Each Index $K, L, I_{1}, I_{2}, I_{3}, I_{4}$ AND $I_{5}$ IN (17) to AChiEve Three Significant Figure AcCuracy when $r_{1}=r_{2}=r_{3}=r$.

\begin{tabular}{|c|c|c|c|c|}
\hline$a$ & $\rho$ & $r=1$ & $r=2$ & $r=5$ \\
\hline 1 & \multirow{2}{*}{0.1} & $2,2,2,2,2,2,3$ & $2,2,3,3,3,3,3$ & $2,2,4,4,4,4,4$ \\
3 & & $3,2,4,4,4,4,4$ & $3,3,5,5,5,5,5$ & $4,4,6,6,6,6,6$ \\
\hline 1 & \multirow{2}{*}{0.3} & $2,2,3,3,3,3,3$ & $2,2,3,3,3,3,3$ & $3,3,4,4,4,4,4$ \\
3 & & $3,3,4,4,4,4,4$ & $4,4,7,7,7,7,7$ & $4,4,7,7,7,7,7$ \\
\hline 1 & \multirow{2}{*}{0.6} & $4,4,4,4,4,4,4$ & $5,5,6,6,6,6,6$ & $6,6,11,11,11,11,11$ \\
3 & & $5,5,6,6,6,6,6$ & $9,9,7,7,7,7,7$ & $11,11,13,13,13,13,13$ \\
\hline
\end{tabular}

of three infinite series of the form

$$
\begin{aligned}
\psi\left(v_{i}\right)= & \sum_{k_{i}=0}^{\infty} \frac{w_{i i}^{2 k_{i}+\frac{n}{2}} a^{2 k_{i}}}{2^{2 k_{i}+\frac{n}{2}} k_{i} !\left(\frac{w_{i i}^{2}}{4}+v_{i}^{2}\right)^{k_{i}+\frac{n}{2}}} \\
& \times \exp \left\{j\left(k_{i}+\frac{n}{2}\right) \arctan \left(\frac{2 v_{i}}{w_{i i}}\right)\right\}, \quad i=1,2,3 .
\end{aligned}
$$

However, the closed-form solution for the chf is not immediately obvious. Nevertheless, it is easy to see that the individual infinite summation is in the form of an exponential series, and with some algebraic manipulation, one can show that the individual chf series reduces to [21, eq.2.1.117].

Before discussing the applications, the truncation error upper bound of (17) is briefly introduced. Let us assume that the infinite series (17) is limited to $K, L, I_{1}, I_{2}, I_{3}, I_{4}$ and $I_{5}$ in the variables $k, l, i_{1}, i_{2}, i_{3}, i_{4}$ and $i_{5}$ respectively. Then the rest of the terms denote the truncation error which can easily be upper bounded using the fact that $\gamma(a, z)<\Gamma(z)$ as shown at the bottom. Further simplification of the upper bound is an arduous task. In fact the former gamma function bound for the incomplete gamma function is not that tight for the most practical purposes. To this end another form of tighter upper bound can be derived following the approach given in Tan and Beaulieu [32] at the expense of more mathematical rigor. Next we shed some light to convergence of the cdf series using numerical figures. For brevity, the number of terms needed in each index in (17) is calculated to achieve three significant figure accuracy, as can be seen from Table III.

\section{APPLICATIONS}

The new results developed in Section III enable the performance analysis of MIMO systems with antenna selection by using space-time block codes in a correlated Rician fading environment. This section presents a bit error rate (BER)

$$
\begin{aligned}
& \psi\left(v_{1}, v_{2}, v_{3}\right)=W_{3}^{\frac{n}{2}} \Gamma^{2}\left(\frac{n-2}{2}\right) \exp \left(-\frac{a^{2} w_{4}}{2}\right) \sum_{k, l=0}^{\infty} \sum_{m=0}^{\min (k, l)}(-1)^{k+l} A(m, k, l) \nu_{k} \nu_{l}\left(\begin{array}{c}
n+\eta_{k l}-3 \\
n-3
\end{array}\right) \\
& \times \sum_{i_{1}, i_{2}, i_{3}, i_{4}, i_{5}=0}^{\infty} \frac{\exp \left\{j \delta_{1} \arctan \left(\frac{2 v_{1}}{w_{11}}\right)+j \delta_{2} \arctan \left(\frac{2 v_{2}}{w_{22}}\right)+j \delta_{3} \arctan \left(\frac{2 v_{3}}{w_{33}}\right)\right\}}{2^{\sum_{j=1}^{5} \lambda_{j}-\frac{3 n}{2}} i_{1} ! i_{2} ! i_{3} ! i_{4} ! i_{5} ! \Gamma\left(i_{1}+\frac{n}{2}+k\right) \Gamma\left(i_{2}+\frac{n}{2}+k\right) \Gamma\left(i_{3}+\frac{n}{2}+l\right)} \\
& \times \frac{a^{\sum_{j=1}^{3} \lambda_{j}} w_{1}^{\lambda_{1}} w_{2}^{\lambda_{2}} w_{3}^{\lambda_{3}} w_{12}^{\lambda_{4}} w_{23}^{\lambda_{5}} \Gamma\left(\delta_{1}\right) \Gamma\left(\delta_{2}\right) \Gamma\left(\delta_{3}\right)}{\Gamma\left(i_{4}+\frac{n}{2}+l\right) \Gamma\left(i_{5}+\frac{n}{2}+k+l-2 m\right)\left(\frac{w_{11}^{2}}{4}+v_{1}^{2}\right)^{\frac{\delta_{1}}{2}}\left(\frac{w_{22}^{2}}{4}+v_{2}^{2}\right)^{\frac{\delta_{2}}{2}}\left(\frac{w_{33}^{2}}{4}+v_{3}^{2}\right)^{\frac{\delta_{3}}{2}}} \\
& E_{T R(c d f)}<\sum_{k=0}^{K-1} \sum_{l=0}^{L-1} \sum_{m=0}^{\min (k, l)} \sum_{i_{1}=0}^{I_{1}-1} \sum_{i_{2}=0}^{I_{2}-1} \sum_{i_{3}=0}^{I_{3}-1} \sum_{i_{4}=0}^{I_{4}-1} \sum_{i_{5}=I_{5}}^{\infty} S_{\epsilon}+\sum_{k=0}^{K-1} \sum_{l=0}^{L-1} \sum_{m=0}^{\min (k, l)} \sum_{i_{1}=0}^{I_{1}-1} \sum_{i_{2}=0}^{I_{2}-1} \sum_{i_{3}=0}^{I_{3}-1} \sum_{i_{4}=I_{4}}^{\infty} \sum_{i_{5}=0}^{\infty} S_{\epsilon} \\
& +\sum_{k=0}^{K-1} \sum_{l=0}^{L-1} \sum_{m=0}^{\min (k, l)} \sum_{i_{1}=0}^{I_{1}-1} \sum_{i_{2}=0}^{I_{2}-1} \sum_{i_{3}=I_{3}}^{\infty} \sum_{i_{4}=0}^{\infty} \sum_{i_{5}=0}^{\infty} S_{\epsilon}+\sum_{k=0}^{K-1} \sum_{l=0}^{L-1} \sum_{m=0}^{\min (k, l)} \sum_{i_{1}=0}^{I_{1}-1} \sum_{i_{2}=I_{2}}^{\infty} \sum_{i_{3}=0}^{\infty} \sum_{i_{4}=0}^{\infty} \sum_{i_{5}=0}^{\infty} S_{\epsilon} \\
& +\sum_{k=0}^{K-1} \sum_{l=0}^{L-1} \sum_{m=0}^{\min (k, l)} \sum_{i_{1}=I_{1}}^{\infty} \sum_{i_{2}=0}^{\infty} \sum_{i_{3}=0}^{\infty} \sum_{i_{4}=0}^{\infty} \sum_{i_{5}=0}^{\infty} S_{\epsilon}+\sum_{k=0}^{K-1} \sum_{l=L}^{\infty} \sum_{m=0}^{\min (k, l)} \sum_{i_{1}=0}^{\infty} \sum_{i_{2}=0}^{\infty} \sum_{i_{3}=0}^{\infty} \sum_{i_{4}=0}^{\infty} \sum_{i_{5}=0}^{\infty} S_{\epsilon} \\
& +\sum_{k=K}^{\infty} \sum_{l=0}^{\infty} \sum_{m=0}^{\min (k, l)} \sum_{i_{1}=0}^{\infty} \sum_{i_{2}=0}^{\infty} \sum_{i_{3}=0}^{\infty} \sum_{i_{4}=0}^{\infty} \sum_{i_{5}=0}^{\infty} S_{\epsilon} \\
& S_{\epsilon}=\frac{W_{3}^{\frac{n}{2}} \Gamma^{2}\left(\frac{n-2}{2}\right) \exp \left(-\frac{a^{2} w_{4}}{2}\right) A(m, k, l) \nu_{k} \nu_{l}\left(\begin{array}{c}
n+\eta_{k l}-3 \\
n-2
\end{array}\right) \nu_{k} \nu_{l} a^{\sum_{j=1}^{3} \lambda_{j}}}{2^{\frac{\sum_{j=1}^{3} \lambda_{j}}{2}} i_{1} ! i_{2} ! i_{3} ! i_{4} ! i_{5} ! \Gamma\left(i_{1}+\frac{n}{2}+k\right) \Gamma\left(i_{2}+\frac{n}{2}+k\right) \Gamma\left(i_{3}+\frac{n}{2}+l\right) \Gamma\left(i_{4}+\frac{n}{2}+l\right)} \\
& \times \frac{w_{1}^{\lambda_{1}} w_{2}^{\lambda_{2}} w_{3}^{\lambda_{3}}\left|w_{12}\right|^{\lambda_{4}}\left|w_{23}\right|^{\lambda_{5}} \Gamma\left(\delta_{1}\right) \Gamma\left(\delta_{2}\right) \Gamma\left(\delta_{3}\right)}{\Gamma\left(i_{5}+\frac{n}{2}+k+l-2 m\right) w_{11}^{\delta_{1}} w_{22}^{\delta_{2}} w_{33}^{\delta_{3}}} .
\end{aligned}
$$


expression for a Gray coded $M$-ary amplitude modulation scheme with OSTBC and transmit antenna selection. A capacity expression is also derived for the same scenario.

The MIMO technology is well known to mitigate the adversary effects of fading and to enhance the capacity of wireless links. The full-diversity order promised by MIMO technology can be exploited by using OSTBC, which utilizes low-complexity maximum-likelihood detection [5]. Nevertheless, full-rate OSTBC do not exist for all transmit antenna configurations. The most celebrated and the only full-rate complex designed OSTBC scheme is known as the Alamouti scheme [33], which employs two transmit antennas. Transmit antenna selection (TAS) [3] is another scheme where a subset of transmit antennas is selected at a particular time. This scheme reduces the amount of hardware, yet retains the diversity benefit. The performance of TAS with OSTBC is analyzed in many papers [34]- [37]. However, no fading correlation is considered in them. In the following, the performance of TAS with OSTBC in the presence of transmit correlation is analyzed.

We consider a MIMO system in a correlated Rician fading environment with 3 transmit and 2 receive antennas. Two transmit antennas out of 3 are selected and activated for the transmission of the Alamouti code, while the remaining transmit antenna is inactive. Let $\widetilde{\mathbf{H}} \in \mathbb{C}^{2 \times 2}$ be a submatrix of the channel matrix $\mathbf{H} \in \mathbb{C}^{2 \times 3}$. The elements of $\mathbf{H}=\left[h_{i j}\right]$, which represent the channel gain between $j$ th transmit and $i$ th receive antenna can be modeled as complex Gaussian variables each having a mean $b / \sqrt{2}$ and variance $\sigma^{2} / 2$ per dimension. Moreover, without loss of generality, $b^{2}+\sigma^{2}=1$ for normalizing the average channel gains. Now, it is clear that $\widetilde{\mathbf{H}}$ consists of the channel gains for the selected two transmit and two receive antennas. Suppose that $\mathbf{h}_{j}(j=1,2,3)$ are the columns of the channel matrix $\mathbf{H}$. The columns are sorted according to their norms such that $\left\|\mathbf{h}_{3}\right\| \geq\left\|\mathbf{h}_{2}\right\| \geq\left\|\mathbf{h}_{1}\right\|$ [34]. Since only transmit correlation is considered, the covariance matrix of $\operatorname{vec}(\mathbf{H}), \mathbf{C}_{\mathbf{H}}$ can be written as

$$
\mathbf{C}_{\mathbf{H}}=\sigma^{2}\left(\begin{array}{ccc}
1 & \rho & \rho^{2} \\
\rho & 1 & \rho \\
\rho^{2} & \rho & 1
\end{array}\right) \otimes \mathbf{I}_{2 \times 2}=\mathbf{R}_{T} \otimes \mathbf{I}_{2 \times 2},
$$

where $\rho^{|j-k|}=\frac{E\left(h_{i j} h_{i k}^{*}\right)-b^{2}}{\sigma^{2}}(i=1,2,3), \mathbf{R}_{T}$ denotes the transmit correlation matrix, $\mathbf{I}_{2 \times 2}$ is the $2 \times 2$ identity matrix, $v e c($.$) is the vectorization operation, \otimes$ denotes the Kronecker product, and $*$ denotes the complex conjugate operation. When the Alamouti code is used, the MIMO system can be replaced with equivalent single input single output (SISO) systems defined as in [5], [34], [35]:

$$
\tilde{s}_{q}=\sqrt{\frac{E_{s}}{2}}\left(\|\tilde{\mathbf{H}}\|_{F}^{2}\right) s_{q}+\tilde{\nu}_{q}, \quad q=1,2,
$$

where $\sqrt{\frac{E_{s}}{2}}$ ensures that the total transmitted power in each channel use is $E_{s}$ and is independent of the number of transmit antennas, $\tilde{s}_{q}, s_{q}$ denote the received and transmitted symbols, $\|\cdot\|_{F}$ is the Frobenius norm, and $\tilde{\nu}_{q}$ is the circular symmetric additive complex Gaussian noise having a zero mean and variance $\frac{\|\widetilde{\mathbf{H}}\|_{F}^{2} N_{0}}{2}$ per dimension.

\section{A. BER Analysis of M-ary PAM Scheme}

The achievable SNR per bit in a M-ary PAM constellation with TAS and the Alamouti scheme can be written as [34]

$$
\gamma_{b}=\frac{\gamma}{2 \log _{2} M}\|\widetilde{\mathbf{H}}\|_{F}^{2}=c \gamma\|\widetilde{\mathbf{H}}\|_{F}^{2},
$$

where $\gamma=\frac{E_{s}}{N_{0}}$ and $c=\frac{1}{2 \log _{2} M}$. Now, it is clear that the antenna-selection criterion mentioned above maximizes the instantaneous SNR.

Let $\gamma_{k}=c \gamma\left\|\mathbf{h}_{k}\right\|^{2}, k=1,2,3$ be the scaled norms of the columns of $\mathbf{H}$. Alternatively, if the $l$ th element of $\mathbf{h}_{k}$ is rewritten as $\left(\Re\left[h_{l k}\right] \Im\left[h_{l k}\right]\right)^{T}$, then the original $\mathbf{H}$ can be written as a $4 \times 3$ matrix, $\mathbf{H}_{e q}$ defined as

$$
\mathbf{H}_{e q}=\left(\begin{array}{ccc}
\Re\left[h_{11}\right] & \Re\left[h_{12}\right] & \Re\left[h_{13}\right] \\
\Im\left[h_{11}\right] & \Im\left[h_{12}\right] & \Im\left[h_{13}\right] \\
\Re\left[h_{21}\right] & \Re\left[h_{22}\right] & \Re\left[h_{23}\right] \\
\Im\left[h_{21}\right] & \Im\left[h_{22}\right] & \Im\left[h_{23}\right]
\end{array}\right),
$$

where $\Re($.$) and \Im($.$) denote the real and imaginary part$ of a complex number. Since only the transmit correlation is considered, the rows of $\mathbf{H}_{e q}$ will be independent and the columns will have the covariance of $\frac{1}{2} \mathbf{R}_{T}$. Then, it is clear that $\gamma_{k}$ are correlated non-central chi-squared random variables having underlying Gaussian variables with a tridiagonal form of inverse covariance matrix. Then the achievable SNR (22) can be written as

$$
\gamma_{b}=\gamma_{2}+\gamma_{3}
$$

Since this SNR is a sum of order statistics, we may follow the approach given in [38] to obtain the joint pdf of $\gamma_{2}, \gamma_{3}$ as (see Appendix A) shown at the bottom, where $y \geq x>0$, $d_{k}=\frac{w_{k k}}{2 c \gamma}, A_{\Sigma}$ is given at the top of the next page, with $2 b^{2}=$ $a^{2}, W_{3}=\frac{8}{\sigma^{6}\left(1-\rho^{2}\right)^{2}}, w_{11}=w_{33}=\frac{2}{\sigma^{2}\left(1-\rho^{2}\right)}, w_{22}=\frac{2\left(1+\rho^{2}\right)}{\sigma^{2}\left(1-\rho^{2}\right)}$, and $w_{12}=w_{23}=\frac{-2 \rho}{\sigma^{2}\left(1-\rho^{2}\right)}$. Using the definition for the Laplace transform, the moment generating function (mgf) of

$$
\begin{aligned}
& f_{\gamma_{2}, \gamma_{3}}(x, y)=A_{\Sigma} \sum_{\substack{u, v, w=1 \\
u \neq v \neq w}}^{3} d_{u}^{\delta_{u}} d_{v}^{\delta_{v}} x^{\delta_{u}-1} y^{\delta_{v}-1} \exp \left\{-\left(d_{u} x+d_{v} y\right)\right\}\left[\left(\delta_{w}-1\right) !-\exp \left(-d_{w} x\right) \sum_{q=0}^{\delta_{w}-1} \frac{\left(\delta_{w}-1\right) !}{q !} d_{w}^{q} x^{q}\right], \\
& \delta_{1}=i_{1}+i_{2}+k+2 \quad \delta_{2}=i_{2}+i_{4}+i_{5}+k+2-m \quad \delta_{3}=i_{3}+i_{4}+l+2 \quad \lambda_{1}=2 i_{1}+k \\
& \begin{array}{llll}
\lambda_{2}=2 i_{5}+k+l-2 m & \lambda_{3}=2 i_{3}+l & \lambda_{4}=2 i_{2}+k & \lambda_{5}=2 i_{4}+l .
\end{array}
\end{aligned}
$$




$$
\begin{aligned}
A_{\Sigma}= & W_{3}^{2} \exp \left(-b^{2} w_{4}\right) \sum_{k, l=0}^{\infty} \sum_{\substack{m=0 \\
\text { min }(k, l)}}^{\infty} \sum_{\substack{i_{1}, i_{2}, i_{3}, i_{4}, i_{5}=0 \\
(k+1)}}^{\infty}(-1)^{k+l} A(m, k, l)\left(\begin{array}{c}
k+l+1-2 m \\
1
\end{array}\right) \\
& \times \frac{b^{\lambda_{1}+\lambda_{2}+\lambda_{3}} w_{1}^{\lambda_{1}} w_{2}^{\lambda_{2}} w_{3}^{\lambda_{3}} w_{12}^{\lambda_{4}} w_{23}^{\lambda_{5}}}{w_{11}^{\delta_{1}} w_{22}^{\delta_{2}} w_{33}^{\delta_{3}} i_{1} ! i_{2} ! i_{3} ! i_{4} ! i_{5}\left(i_{1}+k+1\right) !\left(i_{2}+k+1\right) !\left(i_{3}+l+1\right) !\left(i_{4}+l+1\right) !\left(i_{5}+k+l+1-2 m\right) !}
\end{aligned}
$$

$\gamma_{b}$ can be written as shown in (25). The average BER of an OSTBC with $M$-PAM is given by [34]

$$
P_{M}(\gamma)=\frac{1}{\log _{2} M} \sum_{n=1}^{\log _{2} M} P_{M}(n ; \gamma),
$$

where

$$
P_{M}(n ; \gamma)=\frac{2}{M} \sum_{i=0}^{k_{n}} B_{i}(n) \frac{1}{\pi} \int_{0}^{\infty} \frac{\Phi_{\gamma_{b}}\left(\frac{D_{i}^{2}\left(1+t^{2}\right)}{2}\right)}{1+t^{2}} d t
$$

with

$$
\begin{aligned}
k_{n} & =\left(1-\frac{1}{2^{n}}\right) M-1 \\
B_{i}(n) & =(-1)^{\left\lfloor\frac{i 2^{n-1}}{M}\right\rfloor}\left(2^{n-1}-\left\lfloor\frac{i 2^{n-1}}{M}+\frac{1}{2}\right\rfloor\right) \\
D_{i} & =(2 i+1) \sqrt{\frac{6 \log _{2} M}{M^{2}-1}} .
\end{aligned}
$$

Substituting (25) in (27) and subsequent integration yields the integrals of the form

$$
I\left(\alpha_{1}, \alpha_{2}, m, n\right)=\int_{0}^{\infty} \underbrace{\frac{1}{\left(1+z^{2}\right)\left(\alpha_{1}^{2}+z^{2}\right)^{m}\left(\alpha_{2}^{2}+z^{2}\right)^{n}}}_{f(z)} d z .
$$

These integrals can be solved by evaluating the residues of $f(z)$ in the complex plane as

$$
I(\alpha, \beta, m, n)=\pi j\left[\sum_{i=1}^{2} \operatorname{Res}_{z=j \alpha_{i}} f(z)+\operatorname{Res}_{z=j} f(z)\right],
$$

where $\operatorname{Res}_{z=z_{0}} f(z)$ is the residue of function $f(z)$ evaluated at $z=z_{0}$. Then the BER can be written as shown in (28) at the bottom.

Since (28) has seven fold infinite summations, the numerical evaluation of it would definitely be an arduous task. Hence it is natural to truncate the infinite summations, thereby introducing truncation error in the numerical calculations. Let us assume that the index values $k, l, i_{1}, i_{2}, i_{3}, i_{4}$ and $i_{5}$ are limited to $K, L, I_{1}, I_{2}, I_{3}, I_{4}$ and $I_{5}$ terms respectively. Then the truncation error can be written as shown at the bottom. Furthermore, the number of terms needed in (28) to achieve three significant figure accuracy is given in Table IV. It should be noted that the Rician factor $K$ and $b$ are related as $b=\sqrt{\frac{K}{K+1}}$.

\section{B. Capacity Analysis}

Since equal power allocation is assumed, the average capacity in nats/s/Hz is given by [35], [39]:

$$
C=E\left\{\log \left(1+\gamma_{s}\right)\right\}=\int_{0}^{\infty} \log (1+\lambda) f_{\gamma_{s}}(\lambda) d \lambda,
$$

$$
\begin{aligned}
& \Phi_{\gamma_{b}}(s)= \int_{0}^{\infty} \int_{x}^{\infty} \exp \{-s(x+y)\} f_{\gamma_{2} \gamma_{3}}(x, y) d y d x \\
&=A_{\Sigma} \sum_{\substack{u, v, w=1 \\
u \neq v \neq w}}^{3} d_{u}^{\delta_{u}} d_{v}^{\delta_{v}}\left(\delta_{w}-1\right) !\left(\delta_{v}-1\right) !\left\{\sum_{p=0}^{\delta_{v}-1} \frac{\left(p+\delta_{u}-1\right) !}{p !\left(d_{v}+s\right)^{p+\delta_{v}}\left(d_{u}+d_{v}+2 s\right)^{p+\delta_{u}}}\right. \\
&\left.\quad-\sum_{p=0}^{\delta_{v}-1} \sum_{q=0}^{\delta_{w}-1} \frac{d_{w}^{q}\left(p+q+\delta_{u}-1\right) !}{p ! q !\left(d_{v}+s\right)^{p+\delta_{v}}\left(d_{u}+d_{v}+d_{w}+2 s\right)^{p+q+\delta_{u}}}\right\} .
\end{aligned}
$$

$$
\begin{aligned}
P(\gamma)= & \frac{2}{M \pi \log _{2} M} \sum_{n=1}^{\log _{2} M} \sum_{i=0}^{k_{n}} A_{\Sigma} B_{i}(n) \sum_{\substack{u, v, w=1 \\
u \neq v \neq w}}^{3} 2^{\delta_{v}} d_{u}^{\delta_{u}} d_{v}^{\delta_{v}}\left(\delta_{w}-1\right) !\left(\delta_{v}-1\right) ! \\
& \times\left\{\sum_{p=0}^{\delta_{v}-1} \frac{2^{p}\left(p+\delta_{u}-1\right) !}{p ! D_{i}^{2\left(\delta_{u}+\delta_{v}+2 p\right)}} I\left(\sqrt{\frac{2 d_{v}}{D_{i}^{2}}+1}, \sqrt{\frac{d_{u}+d_{v}}{D_{i}^{2}}+1}, \delta_{v}+p, \delta_{u}+p\right)\right. \\
& \left.-\sum_{p=0}^{\delta_{v}-1} \sum_{q=0}^{\delta_{w}-1} \frac{2^{p} d_{w}^{q}\left(p+q+\delta_{u}-1\right) !}{p ! q ! D_{i}^{2\left(\delta_{u}+\delta_{v}+2 p+q\right)}} I\left(\sqrt{\frac{2 d_{v}}{D_{i}^{2}}+1}, \sqrt{\frac{d_{u}+d_{v}+d_{w}}{D_{i}^{2}}+1}, \delta_{v}+p, \delta_{u}+p+q\right)\right\} .
\end{aligned}
$$


where the achievable SNR per symbol for an M-ary constel- defined as [39, App. B] (for $m=1,2 \ldots$ and $\theta>0$ ) lation, $\gamma_{s}$, is defined as

$$
\gamma_{s}=c \gamma\|\tilde{\mathbf{H}}\|_{F}^{2}
$$

with $c=1 / 2$. Following analogous steps as in (22)-(25) and inverting $\Phi_{\gamma_{s}}\left(\gamma_{s}\right)$ (see Appendix B) and subsequent integration of (29) with $\widehat{C}_{m-1}(\theta)$ yields (31). Note that $\widehat{C}_{m-1}(\theta)$ is

$$
\begin{aligned}
\widehat{C}_{m-1}(\theta) & =\int_{0}^{\infty} \log (1+\lambda) \lambda^{m-1} \exp (-\theta \lambda) d \lambda \\
& =(m-1) ! \exp (\theta) \sum_{k=1}^{m} \frac{\Gamma(-m+k, \theta)}{\theta^{k}}
\end{aligned}
$$

$$
\begin{aligned}
& E_{T R(P(\gamma))}=\frac{2}{M \pi \log _{2} M} \sum_{n=1}^{\log _{2} M} \sum_{i=0}^{k_{n}} \sum_{k=0}^{K-1} \sum_{l=0}^{L-1} \sum_{m=0}^{\min (k, l)} \sum_{i_{1}=0}^{I_{1}-1} \sum_{i_{2}=0}^{I_{2}-1} \sum_{i_{3}=0}^{I_{3}-1} \sum_{i_{4}=0}^{I_{4}-1} \sum_{i_{5}=I_{5}}^{\infty} T_{\epsilon} B_{i}(n) \\
& +\frac{2}{M \pi \log _{2} M} \sum_{n=1}^{\log _{2} M} \sum_{i=0}^{k_{n}} \sum_{k=0}^{K-1} \sum_{l=0}^{L-1} \sum_{m=0}^{\min (k, l)} \sum_{i_{1}=0}^{I_{1}-1} \sum_{i_{2}=0}^{I_{2}-1} \sum_{i_{3}=0}^{I_{3}-1} \sum_{i_{4}=I_{4}}^{\infty} \sum_{i_{5}=0}^{\infty} T_{\epsilon} B_{i}(n) \\
& +\frac{2}{M \pi \log _{2} M} \sum_{n=1}^{\log _{2} M} \sum_{i=0}^{k_{n}} \sum_{k=0}^{K-1} \sum_{l=0}^{L-1} \sum_{m=0}^{m i n(k, l)} \sum_{i_{1}=0}^{I_{1}-1} \sum_{i_{2}=0}^{I_{2}-1} \sum_{i_{3}=I_{3}}^{\infty} \sum_{i_{4}=0}^{\infty} \sum_{i_{5}=0}^{\infty} T_{\epsilon} B_{i}(n) \\
& +\frac{2}{M \pi \log _{2} M} \sum_{n=1}^{\log _{2} M} \sum_{i=0}^{k_{n}} \sum_{k=0}^{K-1} \sum_{l=0}^{L-1} \sum_{m=0}^{\min (k, l)} \sum_{i_{1}=0}^{I_{1}-1} \sum_{i_{2}=I_{2}}^{\infty} \sum_{i_{3}=0}^{\infty} \sum_{i_{4}=0}^{\infty} \sum_{i_{5}=0}^{\infty} T_{\epsilon} B_{i}(n) \\
& +\frac{2}{M \pi \log _{2} M} \sum_{n=1}^{\log _{2} M} \sum_{i=0}^{k_{n}} \sum_{k=0}^{K-1} \sum_{l=0}^{L-1} \sum_{m=0}^{\min (k, l)} \sum_{i_{1}=I_{1}}^{\infty} \sum_{i_{2}=0}^{\infty} \sum_{i_{3}=0}^{\infty} \sum_{i_{4}=0}^{\infty} \sum_{i_{5}=0}^{\infty} T_{\epsilon} B_{i}(n) \\
& +\frac{2}{M \pi \log _{2} M} \sum_{n=1}^{\log _{2} M} \sum_{i=0}^{k_{n}} \sum_{k=0}^{K-1} \sum_{l=L}^{\infty} \sum_{m=0}^{\min (k, l)} \sum_{i_{1}=0}^{\infty} \sum_{i_{2}=0}^{\infty} \sum_{i_{3}=0}^{\infty} \sum_{i_{4}=0}^{\infty} \sum_{i_{5}=0}^{\infty} T_{\epsilon} B_{i}(n) \\
& +\frac{2}{M \pi \log _{2} M} \sum_{n=1}^{\log _{2} M} \sum_{i=0}^{k_{n}} \sum_{k=K}^{\infty} \sum_{l=0}^{\infty} \sum_{m=0}^{\min (k, l)} \sum_{i_{1}=0}^{\infty} \sum_{i_{2}=0}^{\infty} \sum_{i_{3}=0}^{\infty} \sum_{i_{4}=0}^{\infty} \sum_{i_{5}=0}^{\infty} T_{\epsilon} B_{i}(n) \\
& T_{\epsilon}=(-1)^{k+l} W_{3}^{2} \exp \left(-b^{2} w_{4}\right) A(m, k, l)\left(\begin{array}{c}
k+l+1-2 m \\
1
\end{array}\right) \\
& \times \frac{(k+1)(l+1) b^{\lambda_{1}+\lambda_{2}+\lambda_{3}} w_{1}^{\lambda_{1}} w_{2}^{\lambda_{2}} w_{3}^{\lambda_{3}} w_{12}^{\lambda_{4}} w_{23}^{\lambda_{5}}}{w_{11}^{\delta_{1}} w_{22}^{\delta_{2}} w_{33}^{\delta_{3}} i_{1} ! i_{2} ! i_{3} ! i_{4} ! i_{5}\left(i_{1}+k+1\right) !\left(i_{2}+k+1\right) !\left(i_{3}+l+1\right) !\left(i_{4}+l+1\right) !\left(i_{5}+k+l+1-2 m\right) !} \\
& \times \sum_{\substack{u, v, w=1 \\
u \neq v \neq w}}^{3} 2^{\delta_{v}} d_{u}^{\delta_{u}} d_{v}^{\delta_{v}}\left(\delta_{w}-1\right) !\left(\delta_{v}-1\right) !\left\{\sum_{p=0}^{\delta_{v}-1} \frac{2^{p}\left(p+\delta_{u}-1\right) !}{p ! D_{i}^{2\left(\delta_{u}+\delta_{v}+2 p\right)}} I\left(\sqrt{\frac{d_{v}}{D_{i}^{2}}+1}, \sqrt{\frac{d_{u}+d_{v}}{D_{i}^{2}}+1}, \delta_{v}+p, \delta_{u}+p\right)\right. \\
& \left.-\sum_{p=0}^{\delta_{v}-1} \sum_{q=0}^{\delta_{w}-1} \frac{2^{p} d_{w}^{q}\left(p+q+\delta_{u}-1\right) !}{p ! q ! D_{i}^{2\left(\delta_{u}+\delta_{v}+2 p+q\right)}} I\left(\sqrt{\frac{d_{v}}{D_{i}^{2}}+1}, \sqrt{\frac{d_{u}+d_{v}+d_{w}}{D_{i}^{2}}+1}, \delta_{v}+p, \delta_{u}+p+q\right)\right\} .
\end{aligned}
$$

$$
\begin{aligned}
C= & A_{\Sigma} \sum_{\substack{u, v, w=1 \\
u \neq v \neq w}}^{3} d_{u}^{\delta_{u}} d_{v}^{\delta_{v}}\left(\delta_{w}-1\right) !\left(\delta_{v}-1\right) !\left\{\sum _ { p = 0 } ^ { \delta _ { v } - 1 } \frac { ( p + \delta _ { u } - 1 ) ! } { p ! 2 ^ { p + \delta _ { u } } } \left[\sum_{r_{1}=1}^{\delta_{v}+p} \frac{A_{r_{1}}}{\left(r_{1}-1\right) !} \widehat{C}_{r_{1}-1}\left(d_{v}\right)\right.\right. \\
& \left.\sum_{r_{2}=1}^{\delta_{u}+p} \frac{B_{r_{2}}}{\left(r_{2}-1\right) !} \widehat{C}_{r_{2}-1}\left(\frac{d_{u}+d_{v}}{2}\right)\right]-\sum_{p=0}^{\delta_{v}-1} \sum_{q=0}^{\delta_{w}-1} \frac{d_{w}^{q}\left(p+q+\delta_{u}-1\right) !}{p ! q ! 2^{p+q+\delta_{u}}}\left[\sum_{r_{1}=1}^{\delta_{v}+p} \frac{C_{r_{1}}}{\left(r_{1}-\right) !} \widehat{C}_{r_{1}-1}\left(d_{v}\right)\right. \\
& \left.\left.+\sum_{r_{2}=1}^{\delta_{u}+p+q} \frac{D_{r_{2}}}{\left(r_{2}-1\right) !} \widehat{C}_{r_{2}-1}\left(\frac{d_{u}+d_{v}+d_{w}}{2}\right)\right]\right\}
\end{aligned}
$$


TABLE IV

Number of Terms NeEded in EACH index $K, L, I_{1}, I_{2}, I_{3}, I_{4}$ AND $I_{5}$ IN (28) to AChieve Three Significant Figure AcCuracy for BPSK SIGNALING.

\begin{tabular}{|c|c|c|c|c|}
\hline$K$ & $\rho$ & $\gamma=1(d B)$ & $\gamma=5(d B)$ & $\gamma=8(d B)$ \\
\hline 1 & \multirow{2}{*}{0.1} & $3,3,3,3,3,2,2$ & $4,4,4,4,5,5,4$ & $4,4,5,5,5,5,5$ \\
10 & & $3,4,4,4,4,4,4$ & $5,5,4,4,5,5,5$ & $4,4,6,6,6,6,6$ \\
\hline 1 & \multirow{2}{*}{0.3} & $4,4,4,4,4,3,3$ & $5,5,6,6,6,5,5$ & $6,6,7,7,7,6,6$ \\
10 & & $4,4,5,5,5,4,4$ & $6,6,7,7,7,6,6$ & $7,7,9,9,9,7,9$ \\
\hline 1 & \multirow{2}{*}{0.6} & $4,4,6,6,5,6,5$ & $6,6,8,8,7,7,6$ & $7,7,11,11,11,11,10$ \\
10 & & $5,5,7,7,7,6,6$ & $9,9,11,11,11,7,10$ & $11,11,15,15,15,13,13$ \\
\hline
\end{tabular}

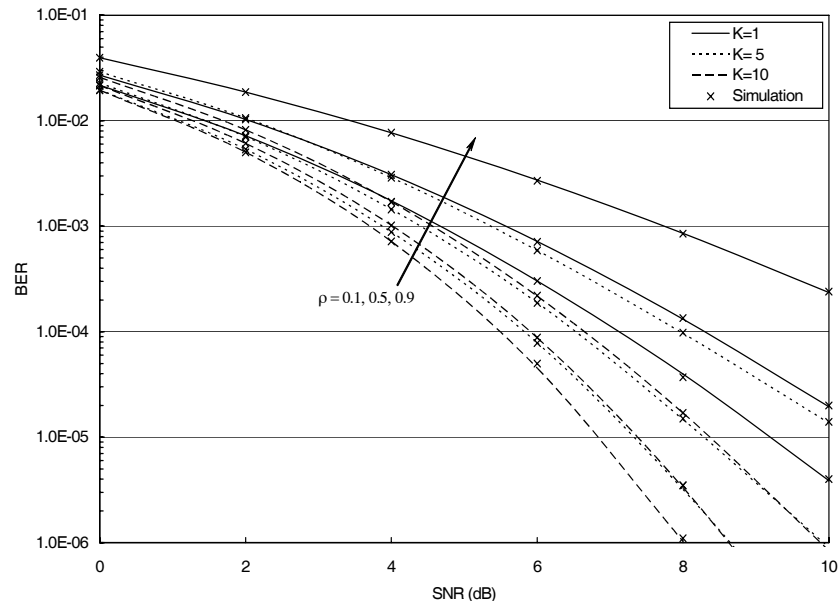

Fig. 1. BER of MIMO with TAS employing Alamouti code and BPSK signaling for different Rician factors ( $K$ values).

$\Gamma(a, z)=\int_{z}^{\infty} \exp (-t) t^{a-1} d t$ is the incomplete complementary gamma function,

$$
\begin{aligned}
A_{r_{1}} & =\left.\frac{1}{\left(\delta_{v}+p-r_{1}\right) !} \frac{\partial^{\delta_{v}+p-r_{1}}}{\partial s^{\delta_{v}+p-r_{1}}}\left[f_{1}(s)\right]\right|_{s=-d_{v}}, \\
B_{r_{2}} & =\left.\frac{1}{\left(\delta_{u}+p-r_{2}\right) !} \frac{\partial^{\delta_{u}+p-r_{2}}}{\partial s^{\delta_{u}+p-r_{2}}}\left[f_{1}(s)\right]\right|_{s=-\left(d_{u}+d_{v}\right)}, \\
C_{r_{1}} & =\left.\frac{1}{\left(\delta_{v}+p-r_{1}\right) !} \frac{\partial^{\delta_{v}+p-r_{1}}}{\partial s^{\delta_{v}+p-r_{1}}}\left[f_{2}(s)\right]\right|_{s=-d_{v}}, \\
D_{r_{2}} & =\frac{\left.\frac{\partial^{\delta_{u}+p+q-r_{2}}}{\partial s^{\delta} u+p+q-r_{2}}\left[f_{2}(s)\right]\right|_{s=-\left(d_{u}+d_{v}+d_{w}\right)}}{\left(\delta_{u}+p+q-r_{2}\right) !}
\end{aligned}
$$

and

$$
\begin{aligned}
& f_{1}(s)=\frac{1}{\left(d_{v}+s\right)^{\delta_{v}+p}\left(\frac{d_{u}+d_{v}}{2}+s\right)^{\delta_{u}+p}}, \\
& f_{2}(s)=\frac{1}{\left(d_{v}+s\right)^{\delta_{v}+p}\left(\frac{d_{u}+d_{v}+d_{w}}{2}+s\right)^{\delta_{u}+p+q}} .
\end{aligned}
$$

Figure 1 depicts the BER performance of MIMO with TAS by using the Alamouti scheme and binary phase shift keying (BPSK) signaling over correlated Rician fading channels. The BPSK signaling is used to reduce the complexity of the evaluation of (28) numerically for different SNR values. As the graph reveals, the fading correlation among the transmit antennas generally downgrades the performance. However, a strong line of sight path between the transmitter and receiver improves the performance.

Figure 2 shows the capacity of MIMO with TAS by using the Alamouti scheme and BPSK signaling over Rician fading

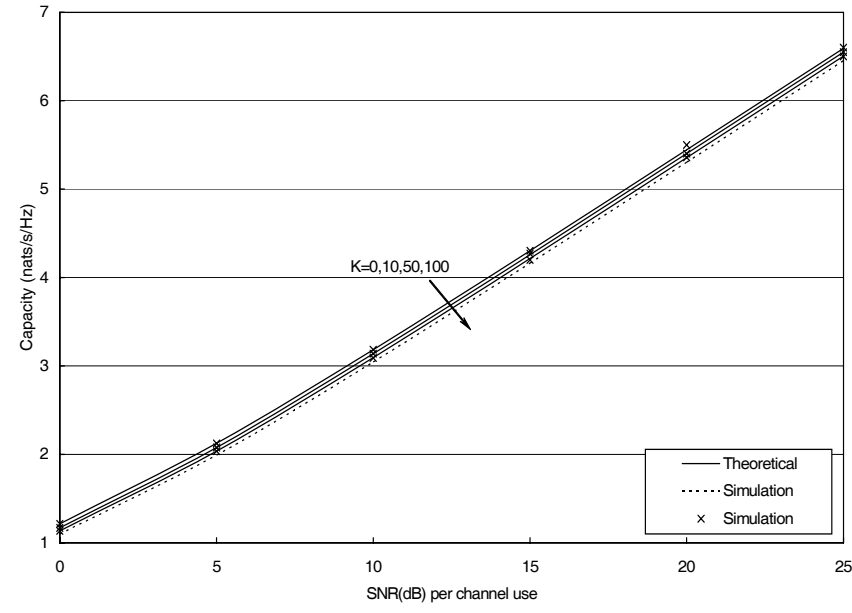

Fig. 2. Capacity of MIMO with TAS employing Alamouti code and BPSK signaling for different Rican factors ( $K$ values).

channels having different Rician factors ( $K$ values). The results are analogous to those given in [40 Fig. 3] for Rician channels with a fixed gain. Since the Rician fading scenario is an intermediate state between the Rayleigh fading (i.e. $K=0$ ) and the deterministic channel (i.e. $K=\infty$ ), a fixed channel gain increase in the $K$ value decreases the amount of scattering. This result is highly undesirable in a MIMO system [40]. The effect of the correlation is not shown here because the curves cannot be distinguished in the figure. Nevertheless, a performance degradation can be observed with the increased correlation even in this scenario.

\section{CONCLUSION}

A new infinite series representation for the trivariate noncentral $\chi^{2}$ pdf has been derived for the case when the underlying Gaussian components have the tridiagonal form of an inverse covariance matrix. An identity for a product of two ultraspherical polynomials, based on Dougall's identity and Miller's approach, is used in the derivation. Moreover, the chf and cdf series are also derived. Some special cases of the joint pdf are also discussed. However, the derivation for an arbitrary covariance matrix seems intractable when using this approach. Furthermore, two applications, namely the analysis of the BER and the capacity of MIMO with TAS and Alamouti coding over correlated Rician fading channels, are considered.

\section{ACKNOWLEDGMENT}

The authors would like to thank the anonymous reviewers for their critical comments that greatly improved this paper. 
The first author would like to thank the government of Finland and the former AIT-Finnish project director late Prof. A. B. Sharma for the doctoral scholarship provided to him.

\section{APPENDIX A \\ DERIVATION OF (24)}

Since $\gamma_{1} \leq \gamma_{2} \leq \gamma_{3}$, following [38] we can write the cdf of $\gamma_{2}$ and $\gamma_{3}$ using the first principles as

$$
\begin{aligned}
F_{\gamma_{2}, \gamma_{3}}(\alpha, \beta)= & \operatorname{Pr}\left(\gamma_{1} \leq \beta, \gamma_{2} \leq \alpha, \gamma_{3} \leq \alpha\right) \\
& +\operatorname{Pr}\left(\gamma_{1} \leq \alpha, \gamma_{2} \leq \beta, \gamma_{3} \leq \alpha\right) \\
& +\operatorname{Pr}\left(\gamma_{1} \leq \alpha, \gamma_{2} \leq \alpha, \gamma_{3} \leq \beta\right) \\
& -2 \operatorname{Pr}\left(\gamma_{1} \leq \alpha, \gamma_{2} \leq \alpha, \gamma_{3} \leq \alpha\right)
\end{aligned}
$$

where $\beta \geq \alpha>0$. Now it can easily be rewritten using (17) as

$$
\begin{aligned}
F_{\gamma_{2}, \gamma_{3}}(\alpha, \beta)= & G\left(\frac{\beta}{c \gamma}, \frac{\alpha}{c \gamma}, \frac{\alpha}{c \gamma}\right)+G\left(\frac{\alpha}{c \gamma}, \frac{\beta}{c \gamma}, \frac{\alpha}{c \gamma}\right) \\
& +G\left(\frac{\alpha}{c \gamma}, \frac{\alpha}{c \gamma}, \frac{\beta}{c \gamma}\right)-2 G\left(\frac{\alpha}{c \gamma}, \frac{\alpha}{c \gamma}, \frac{\alpha}{c \gamma}\right) .
\end{aligned}
$$

Successive differentiation of (34) with respect to $\alpha$ and $\beta$ yields the joint pdf as

$$
\begin{aligned}
\frac{\partial^{2} F_{\gamma_{2}, \gamma_{3}}(\alpha, \beta)}{\partial \alpha \partial \beta}= & f_{\gamma_{2}, \gamma_{3}}(\alpha, \beta) \\
= & A \sum_{\substack{u, v, w=1 \\
u \neq v \neq w}}^{3} d_{u}^{\delta_{u}} d_{v}^{\delta_{v}} \alpha^{\delta_{u}-1} \beta^{\delta_{v}-1} \\
& \times \exp \left\{-\left(d_{u} \alpha+d_{v} \beta\right)\right\} \gamma\left(\delta_{w}, d_{w} \alpha\right) .
\end{aligned}
$$

Since $\delta_{w}$ is an integer, we can express the incomplete gamma function term with the following finite series representation

$$
\begin{aligned}
& \gamma\left(\delta_{w}, d_{w} \alpha\right) \\
& \quad=\left(\delta_{w}-1\right) !-\exp \left(-d_{w} \alpha\right) \sum_{k=0}^{\delta_{w}-1} \frac{\left(\delta_{w}-1\right) !\left(d_{w} \alpha\right)^{k}}{k !} .
\end{aligned}
$$

Substitution of (36) in (35) gives (24).

\section{APPENDIX B \\ DERIVATION OF (31)}

Before we start with the derivation of (31) the following Laplace transform pair is worth of mentioning

$$
\begin{aligned}
\frac{1}{(s+a)^{m}(s+b)^{n}} \longrightarrow & \sum_{r_{1}=1}^{m} A_{r_{1}} \frac{\exp (-a t) t^{r_{1}-1}}{\left(r_{1}-1\right) !} \\
& +\sum_{r_{2}=1}^{n} B_{r_{2}} \frac{\exp (-b t) t^{r_{2}-1}}{\left(r_{2}-1\right) !}
\end{aligned}
$$

where

$$
\begin{aligned}
A_{r_{1}} & =\left.\frac{1}{\left(m-r_{1}\right) !} \frac{\partial^{m-r_{1}}[F(s)]}{\partial s^{m-r_{1}}}\right|_{s=-a}, \\
B_{r_{2}} & =\left.\frac{1}{\left(n-r_{2}\right) !} \frac{\partial^{n-r_{2}}[F(s)]}{\partial s^{n-r_{2}}}\right|_{s=-b} .
\end{aligned}
$$

Now we can make use of (37) in (25) (of course now $c=\frac{1}{2}$ ) to obtain the inverse Laplace transform to yield the pdf of $f_{\gamma_{s}}(\lambda)$ as shown in (38). Then we substitute (38) in (29) and subsequent integration using (32) gives (31).

\section{REFERENCES}

[1] M. K. Simon and M.-S. Alouini, "On the difference of two chisquared variates with application to outage probability computation," IEEE Trans. Commun., vol. 49, no. 11, pp. 1946-1954, Nov. 2001.

[2] G. J. Foschini and Gans M, "On the limit of wireless communication in a fading environment when using multiple antennas," Wireless Pers. Commun., vol. 6, pp. 311-335, Mar. 1998.

[3] D. A. Gore, R. W. Heath, and A. J. Paulraj, "Transmit selection in spatial multiplexing systems," IEEE Commun. Lett., vol. 6, no. 11, pp. 491-493, Nov. 2002.

[4] M. K. Simon and M.-S. Alouini, Digital Communication over Fading Channels. John Wiley \& Sons, Inc., 2005.

[5] H. Jafarkhani, Space-Time Coding. Cambridge University Press, 2005.

[6] T. Cacoullos and M. Koutras, "Quadratic forms in spherical random variables: Generalized non-central $\chi^{2}$ distribution," Naval Res. Logist. Quart., vol. 31, pp. 447-461, 1984.

[7] M. Koutras, "On the generalized noncentral chi-squared distribution induced by elliptical gamma law," Biometrika, vol. 73, pp. 528-532, 1986.

[8] P. B. Patnaik, "The non-central $\chi^{2}$ and F-distributions and their applications," Biometrika, vol. 36, pp. 202-232, 1949.

[9] M. L. Tiku, "Laguerre series forms of non-central $\chi^{2}$ and Fdistributions," Biometrika, vol. 52, pp. 415-427, 1965.

[10] K. H. Biyari and W. C. Lindsey, "Statistical distributions of Hermitian quadratic forms in complex Gaussian variables," IEEE Trans. Inform. Theory, vol. 39, no. 3, pp. 1076-1082, May. 1993.

[11] M. Hagedorn, P. J. Smith, P. J. Bones, R. P. Millane, and D. Pairman, "A trivariate chi-squared distribution derived from the complex Wishart distribution," J. Multivariate Anal., vol. 97, pp. 655-674, 2006.

[12] D. R. Jensen, "The joint distribution of traces of Wishart matrices and some applications," Ann. Math. Stat., vol. 41, pp. 133-145, 1970.

[13] P. R. Krishnaiah, P. Hagis, and L. Steinberg, "A note on the bivariate chi distribution," SIAM Rev., vol. 5, pp. 140-144, 1963.

[14] T. Royen, "Expansions for the multivariate chi-squared distribution," $J$. Multivariate Anal., vol. 38, pp. 213-232, 1991.

[15] L. E. Blumenson and K. S. Miller, "Properties of generalized Rayleigh distributions," Ann. Math. Statis., vol. 34, pp. 903-910, 1963.

[16] K. S. Miller, Multidimensional Gaussian Distributions. John Wiley, 1964.

[17] R. K. Mallik, "On multivariate Rayleigh and exponential distributions," IEEE Trans. Inform. Theory, vol. 49, no. 6, pp. 1499-1515, June 2003.

[18] G. K. Karagiannidis, D. A. Zogas, and S. A. Kotsopoulos, "On the multivariate Nakagami- $m$ distribution with exponential correlation," IEEE Trans. Commun., vol. 51, no. 8, pp. 1240-1244, Aug. 2003.

$$
\begin{aligned}
f_{\gamma_{s}}(\lambda)= & A \sum_{\substack{u, v, w=1 \\
u \neq v \neq w}}^{3} d_{u}^{\delta_{u}} d_{v}^{\delta_{v}}\left(\delta_{w}-1\right) !\left(\delta_{v}-1\right) !\left\{\sum _ { p = 0 } ^ { \delta _ { v } - 1 } \frac { ( p + \delta _ { u } - 1 ) ! } { p ! 2 ^ { p + \delta _ { u } } } \left[\sum_{r_{1}=1}^{\delta_{v}+p} \frac{A_{r_{1}}}{\left(r_{1}-1\right) !} \exp \left(-d_{v} \lambda\right) \lambda^{r_{1}-1}\right.\right. \\
& \left.\times \sum_{r_{2}=1}^{\delta_{u}+p} \frac{B_{r_{2}}}{\left(r_{2}-1\right) !} \exp \left(-\frac{d_{u}+d_{v}}{2} \lambda\right) \lambda^{r_{2}-1}\right]-\sum_{p=0}^{\delta_{v}-1} \sum_{q=0}^{\delta_{w}-1} \frac{d_{w}^{q}\left(p+q+\delta_{u}-1\right) !}{p ! q ! 2^{p+q+\delta_{u}}} \\
& \left.\times\left[\sum_{r_{1}=1}^{\delta_{v}+p} \frac{C_{r_{1}}}{\left(r_{1}-1\right) !} \exp \left(-d_{v} \lambda\right) \lambda^{r_{1}-1}+\sum_{r_{2}=1}^{\delta_{u}+p+q} \frac{D_{r_{2}}}{\left(r_{2}-1\right) !} \exp \left(-\frac{d_{u}+d_{v}+d_{w}}{2} \lambda\right) \lambda^{r_{2}-1}\right]\right\} .
\end{aligned}
$$


[19] G. K. Karagiannidis, D. A. Zogas, and S. A. Kotsopoulos, "An efficient approach to multivariate Nakagami- $m$ distribution using Green's matrix approximation," IEEE Trans. Wireless Commun., vol. 2, no. 5, pp. 883889, Sept. 2003.

[20] K. D. P. Dharmawansa, R. M. A. P. Rajatheva, and C. Tellambura, "Infinite series representations of the trivariate and quadrivariate Nakagamim distributions," IEEE Trans. Wireless Commun., vol. 6, no. 12, pp. 4320-4328, Dec. 2007.

[21] J. G. Proakis, Digital Communications, 3rd ed. McGraw-Hill, 1995.

[22] D. Middleton, An Introduction to Statistical Communication Theory. New York: McGraw-Hill, 1960.

[23] D. A. Zogas and G. K. Karagiannidis, "Infinite-series representations associated with the bivariate Rician distribution and their applications," IEEE Trans. Commun., vol. 53, no. 11, pp. 1790-1794, Nov. 2005.

[24] M. K. Simon, "Comments on "Infinite-series representations associated with the bivariate Rician distribution and their applications"," IEEE Trans. Commun., vol. 54, no. 8, pp. 1511-1512, Aug. 2006.

[25] K. D. P. Dharmawansa, R. M. A. P. Rajatheva, and K. M. Ahmed, "On the bivariate and trivariate Rician distributions," in Proc. IEEE VTC 2006, pp. 1-5, Sept. 25-28.

[26] A. T. James, "The non-central Wishart distribution," Proc. Royal Society of London. Series A, Mathematical and Physical Sciences, vol. 229, no. 1178, pp. 364-366, 1955.

[27] G. E. Andrews, R. Askey, and R. Roy, Special Functions, vol. 71. Cambridge University Press, 1999.

[28] V. A. Aalo, "Performance of maximal-ratio diversity systems in a correlated Nakagami-fading environment," IEEE Trans. Commun., vol. 43, no. 8, pp. 2360-2369, Aug. 1995.

[29] G. N. Watson, A Treatise on the Theory of Bessel Functions. Cambridge, U.K.: Cambridge Univ. Pres, 1944.

[30] I. S. Gradshteyn and I. M. Ryzhik, Tables of Integrals, Series and Products, 5th ed. New York: Academic Press, 1994.

[31] A. Papoulis and S. U. Pillai, Probability, Random Variables and Stochastic Processes, 4th ed. New York: McGraw-Hill, 2002.

[32] C. C. Tan and N. C. Beaulieu, "Infinite series representations of the bivariate Rayleigh and Nakagami- $m$ distributions," IEEE Trans. Commun., vol. 45, no. 10, pp. 1159-1161, Oct. 1997.

[33] S. M. Alamouti, "A simple transmit diversity technique for wireless communications," IEEE J. Select. Areas Commun., vol. 16, no. 8, pp. 1451-1458, Oct. 1998.

[34] S. Kaviani and C. Tellambura, "Closed-form BER analysis for antenna selection using orthogonal space-time block codes," IEEE Commun. Lett., vol. 10, no. 10, pp. 704-706, Oct. 2006.

[35] P. T. Khoa and C. Tellambura, "Capacity analysis for transmit antenna selection using orthogonal space-time block codes," IEEE COmmun. Lett., vol. 11, no. 5, pp. 423-425, May 2007.

[36] X. Cai and G. B. Giannakis, "Performance analysis of combined transmit selection diversity and receive generalized selection combining in Rayleigh fading channels," IEEE Trans. Commun., vol. 3, no. 6, pp. 1980-1983, Nov. 2004.

[37] Z. Chen, J. Yuan, B. Vucetic, and Z. Zhou, "Performance of Alamouti scheme with transmit antenna selection" Electron. Lett., vol. 39, no. 23, pp. 1666-1668, Nov. 2003.

[38] Y. Chen and C. Tellambura, "Infinite series representations of the trivariate and quadrivariate Rayleigh distribution and their applications," IEEE Trans. Commun., vol. 53, no. 12, pp. 2092-2101, Dec. 2005.
[39] M. -S. Alouini and A. J. Goldsmith, "Capacity of Rayleigh fading channels under different adaptive transmission and diversity-combining techniques," IEEE Trans. Veh. Technol., vol. 48, no. 4, pp. 1165-1181, July. 1999.

[40] M. Kang and M.-S. Alouini, "Capacity of MIMO Rician channels," IEEE Trans. Wireless Commun., vol. 5, no. 1, pp. 112-122, Jan. 2006.

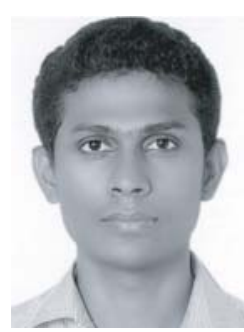

Prathapasinghe Dharmawansa (S'05-M'08) received the B.Sc. and M.Sc. degrees in Electronic and Telecommunication Engineering from the University of Moratuwa, Moratuwa, Sri Lanka in 2003 and 2004 respectively, and the D.Eng. degree in Information and Communications Technologies from the Asian Institute of Technology, Thailand in 2007. Currently he is a Postdoctoral Research Fellow with the Department of Electronic and Computer Engineering, Hong Kong University of Science and Technology, Hong Kong. His research interests are in MIMO systems, random matrix theory and multivariate statistics.

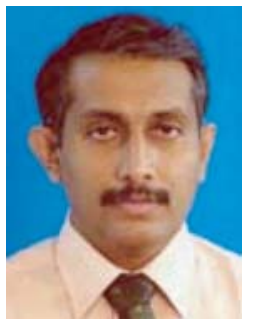

Nandana Rajatheva (SM'01) received the B.Sc degree in Electronic and Telecommunication Engineering (with first- class honors) from the university of Moratuwa, Moratuwa, Sri Lanka, in 1987, and the M.Sc., Ph.D. degrees from the University of Manitoba, Winnipeg, MB, Canada in 1991 and 1995 respectively.

Currently he is an Associate Professor with the Telecommunications Field of Study, School of Engineering and Technology, Asian Institute of Technology (AIT), Thailand. He was with the University of Moratuwa, Sri Lanka before joining AIT where he was promoted to Professor in Electronic \& Telecommunication Engineering in June 2003. From May 1996 to Dec.2001, he was with TC-SAT as an Associate Professor. His research interests include mobile and wireless communications, coding and modulation techniques, space time processing for MIMO systems and communication theory.

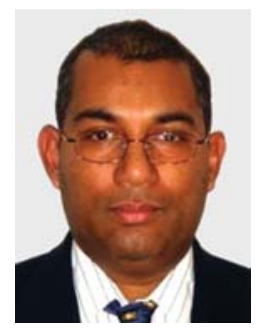

Chintha Tellambura (SM'02) received the B.Sc. degree (with first-class honors) from the University of Moratuwa, Moratuwa, Sri Lanka, in 1986, the M.Sc. degree in electronics from the University of London, London, U.K., in 1988, and the Ph.D. degree in electrical engineering from the University of Victoria, Victoria, BC, Canada, in 1993.

He was a Postdoctoral Research Fellow with the University of Victoria (1993-1994) and the University of Bradford (1995-1996). He was with Monash University, Melbourne, Australia, from 1997 to 2002. Presently, he is a Professor with the Department of Electrical and Computer Engineering, University of Alberta. His research interests include coding, communication theory, modulation, equalization, and wireless communications.

Prof. Tellambura is an Associate Editor for both the IEEE TRANSACTIONS ON COMMUNICATIONS and the IEEE TRANSACTIONS ON WIRELESS COMMUNiCATIONS. He was Chair of the Communication Theory Symposium in Globecom'05 held in St. Louis, MO. 